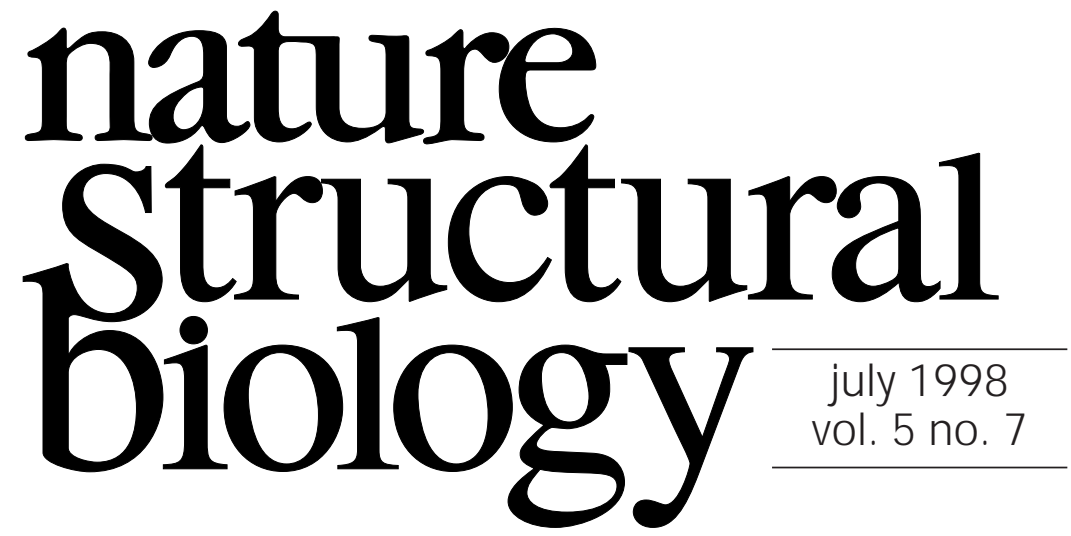

\title{
A new spin on the Web
}

The full text of Nature Structural Biology will — for the first time — be available on-line to subscribers this month.

The first reaction of many will (understandably) be: "At last!". It is certain that implementing electronic delivery of Nature Structural Biology has been slow compared to many other journals in this and related fields. For an area of research where the core readers are quite so computer literate and have access to state-of the-art computing facilities, this could have been a missed opportunity. Luckily, the lack of full text on-line has not had an appreciable negative impact on the journal or its

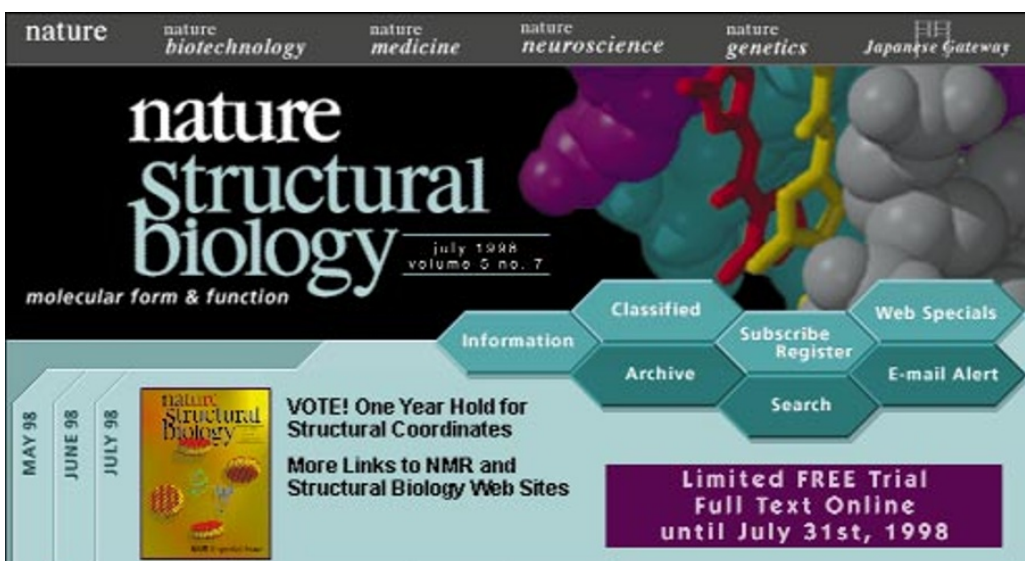

W Copyright 1998 Nature America inc. Site Map Feedback perception in the field. This is perhaps because the functionality and utility of the World Wide Web (or Web, for short) has not yet advanced much beyond being a paper surrogate, at least with respect to individual journals; that is, searching the available contents of a particular journal on the web is presently only moderately faster and more convenient than sitting down with hard-copy volumes of the journal (and, advocates of browsing might argue, less interesting or rewarding).

Why is this so? Where the Web does excel (at the moment), in terms of publishing and disseminating reports of scientific research, is in searching and linking together a much broader range of content than is found in one or even several journals (and delivering new content promptly). One of the ingredients that is still lacking, though, is accessibil-

The home page of the new Nature Structural Biology Website: http://structbio.nature.com. The full content of the site is free during the month of July. ity to all the information that can be located by searching and following links. A would-be internetist is very likely to run up against a register/subscribe window while in pursuit of that all-important reference: it would be prohibitively expensive for someone to subscribe to all the journals that $\mathrm{s} /$ he might encounter in a search of the Web on a particular subject, and it is not yet possible to access and pay for 'one-off use' of documents on many sites.

While publishing companies are exploring and experimenting with models for selling access to both individuals and libraries there are as yet no tried and tested models for publishing high-quality (read, carefully selected and edited) content on the Web and recovering costs, let alone making a profit. Few journals have the luxury of giving away their product (unless they have invested little or nothing in it, in which case it is probably of little value, anyway, or have alternative revenue streams that cover the cost of such philanthropy). At the same time libraries are struggling with ever-tightening budgets, in the face of a flood of new titles competing for space along-side more established publications. Most often, the decisions that librarians have to weigh are which journals to cut from their collections.

There is another fear. More ambitious journals put considerable effort into developing attractive academic and commercial personaes in order to win in the competition for the best papers and top advertising dollars. Yet the Web has the potential of robbing them of their identity. Searches and links have the power to bypass all the ephemera of a hard-copy journal — the cover, the table of 


\section{editorial}

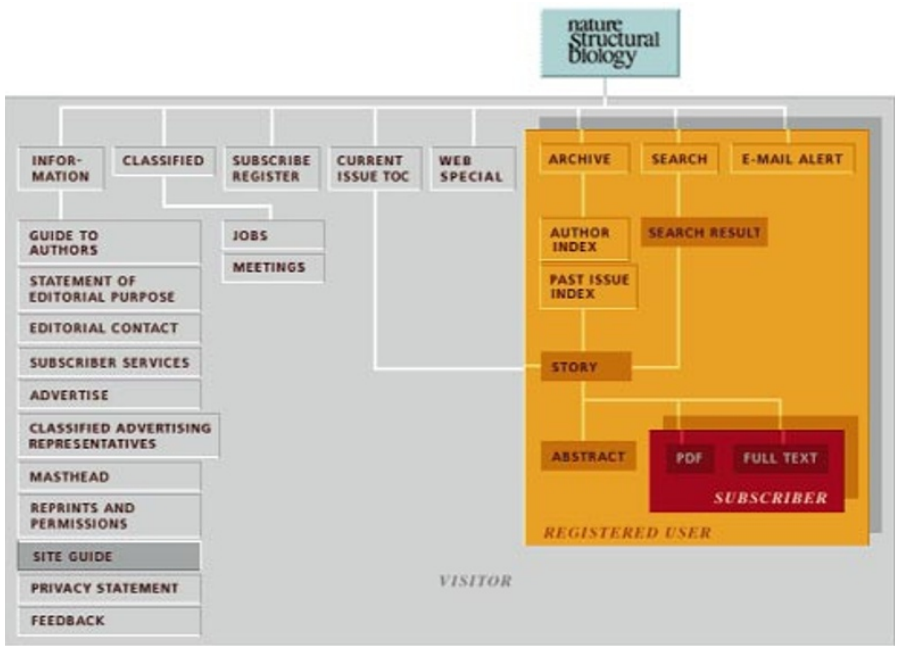

Don't panic: the site guide will take you there.

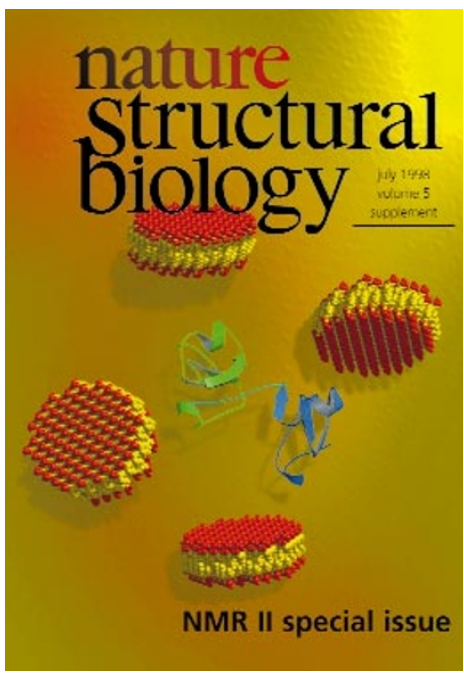

1. NMR II supplement, Nature Struct. Biol. 5,

2. Bewley, C.A. et al. Nature Struct. Biol. 5, 571-578 (1998).

3. Rubinstenn, G. et al. Nature Struct. Biol. 5 568-570 (1998).

\section{Second NMR special issue} proteins. contents, the reviews section ... all those things that help mark out one journal from another - and take the internetist straight to the target of their enquiries.

These problems will inevitably be resolved, perhaps at the relatively cheap cost (for the publishing companies, at least) of the demise of some of the weaker, slower journals or, more radically, a drastic reorganization in the way science is disseminated. Nevertheless, such hurdles - along with the continual but incremental improvement in Web-related technologies have prevented any particular publisher from gaining an unassailable (or even significant) lead in the electronic dissemination of scientific research. Even so, to have delayed for much longer would have, we believe, started to damage our reputation in the eyes of our readers and authors, for the simple reason that the journal would not be as useful as those of our competitors.

What those who explore the site will find is the first iteration of our plans for electronic dissemination of the content of Nature Structural Biology. There are three areas available to the user: a visitor area; a registered user area; and a subscriber area. The details of what is available to each type of user is outlined in the Site Guide. Briefly, visitors have access to information about the journal and the current table of contents. Registered users have access to the above as well as the titles and abstracts of the journal archive (by the end of the month), a search facility, the option of receiving e-mail alerts, and Web-based editorial features. Subscribers to the hard copy version of the journal have access to the above as well as the full text of the journal and supplements, such as the July, 1998, NMR II ${ }^{1}$ and August, 1998, synchrotron radiation supplements. For the first month after the launch, the subscriber-only content will be freely available to all who care to register.

What of the future? Given the nature of the research we publish, it would seem obvious to develop the graphical potential of the Web to allow users of the site to interact more directly with the structures themselves. Perhaps there is an opportunity to utilize the Chime-based 'scripts' that are being used increasingly as teaching tools for structural biology. There is also the opportunity to develop a broader, more integrated Nature Journals site that would cater to the increasingly interdisciplinary nature of scientific research. And, of course, the technology of the Web itself will drive as yet unforeseen innovation: there is much to look forward to.

One of the features available on the new Nature Structural Biology Web site will be (in addition to the July issue) the full-text of the second NMR supplement ${ }^{1}$. Why a second NMR supplement? Part of the answer is provided by Kurt Wüthrich, who notes that roughly one fifth of the structures determined during the period 1990-1996 were obtained using NMR spectroscopy. Iain Campbell and Kristina Downing provide insight into why NMR has been so successful as a structure determination tool, particularly in studying the abundant, (relatively) small modular domains that many proteins are comprised of.

The challenge for structural biomolecular NMR in the future will be its application to bigger, more complex and less tractable systems. James Prestegard and Robert Griffin review the next wave of NMR experiments designed to meet this challenge: Prestegard considers the new orientational data (such as residual dipolar coupling and chemical shift anisotropy) beginning to be used in structure determinations (for an example, see the paper from Gronenborn and colleagues in this issue ${ }^{2}$ ) and Griffin looks at the potential of solid-state NMR techniques in tackling systems such as protein aggregates (prions, amyloid, and so on) and membrane

These areas alone are justification for once again highlighting the technology, but NMR is more than just a means to obtain a collection of structures. An important theme of the series of reviews in the supplement is the power of the technology to probe those macromolecular functions that are not addressed by the beautiful but, for the most part static, images of structures generated by X-ray crystallography: for example, equilibrium (Jane Jyson and Peter Wright) and kinetic (Chris Dobson and Peter Hore) protein folding studies and protein dynamics (Lewis Kay; see Rubinstenn et al. in this issue ${ }^{3}$ ).

What will be the next quantum leap for biomolecular NMR? It is difficult to say for such a young technology. But whatever it may be, perhaps, as Wüthrich suggests, it will be fuelled by a move away from the present cottage industry model of NMR research to an era of 'big science' NMR, in which large 'NMR parks' service hundreds of scientists, in an analogous manner to synchrotron radiation facilities. 